PROCEEDINGS OF THE

AMERICAN MATHEMATICAL SOCIETY

Volume 137, Number 6, June 2009, Pages 2085-2092

S 0002-9939(09)09723-8

Article electronically published on January 22, 2009

\title{
ON GRADIENT RICCI SOLITONS WITH SYMMETRY
}

\author{
PETER PETERSEN AND WILLIAM WYLIE
}

(Communicated by Richard A. Wentworth)

\begin{abstract}
We study gradient Ricci solitons with maximal symmetry. First we show that there are no nontrivial homogeneous gradient Ricci solitons. Thus, the most symmetry one can expect is an isometric cohomogeneity one group action. Many examples of cohomogeneity one gradient solitons have been constructed. However, we apply the main result in our paper "Rigidity of gradient Ricci solitons" to show that there are no noncompact cohomogeneity one shrinking gradient solitons with nonnegative curvature.
\end{abstract}

\section{INTRODUCTION}

The goal of the paper is to study how symmetries can yield rigidity of a gradient Ricci soliton under weaker conditions than those used in 21. Recall that a Ricci soliton is a Riemannian metric together with a vector field $(M, g, X)$ that satisfies

$$
\operatorname{Ric}+\frac{1}{2} L_{X} g=\lambda g
$$

It is called shrinking when $\lambda>0$, steady when $\lambda=0$, and expanding when $\lambda<0$. In the case where $X=\nabla f$, the equation can also be written as

$$
\operatorname{Ric}+\operatorname{Hess} f=\lambda g
$$

and is called a gradient (Ricci) soliton. A gradient soliton is rigid if it is isometric to a quotient of $N \times \mathbb{R}^{k}$, where $N$ is an Einstein manifold, and $f=\frac{\lambda}{2}|x|^{2}$ on the Euclidean factor. Throughout this paper we will also assume that our metrics have bounded curvature. Shi's estimates for the Ricci flow then imply that all the derivatives of curvature are also bounded (see Chapter 6 of [6]).

First we show that all gradient solitons with maximal symmetry are rigid.

Theorem 1.1. All homogeneous gradient Ricci solitons are rigid.

This is in sharp contrast to the more general Ricci solitons that exist on many Lie groups and other homogeneous spaces; see [1, 14, 15]. It also shows that the maximal amount of symmetry we can expect on a nontrivial gradient soliton is a cohomogeneity 1 action that leaves $f$ invariant. Particular cases, such as the rotationally symmetric case on $\mathbb{R}^{n}$ and the $U(n)$ invariant case on certain Kähler manifolds, have been studied extensively, and many interesting examples have been found; see e.g. 4, 5, 7, 9, 11, 12, 13, 23, 27. In particular, Kotschwar 13. has shown that the only rotationally symmetric shrinking gradient soliton metrics on

Received by the editors October 12, 2007, and, in revised form, August 5, 2008.

2000 Mathematics Subject Classification. Primary 53C20.

(C)2009 American Mathematical Society Reverts to public domain 28 years from publication 
$S^{n}, \mathbb{R}^{n}$, and $S^{n-1} \times \mathbb{R}$ are the rigid ones; and Feldman, Ilmanen, and Knopf 9 ] have proven that the only $U(n)$ invariant shrinking soliton on $\mathbb{C}^{n}$ is the flat metric. No curvature assumption is required for these results. On the other hand, there are nonrigid complete noncompact $U(n)$ invariant gradient shrinking solitons [7, 9, 27. These examples show that some other assumption is necessary in general to prove rigidity. Here we show that nonnegative curvature suffices.

Theorem 1.2. All complete noncompact shrinking gradient solitons of cohomogeneity 1 with nonnegative Ricci curvature and $\sec (E, \nabla f) \geq 0$ are rigid.

Recently Naber [16, building on work of Ni and Wallach [19, has shown that every 4-dimensional complete shrinking soliton with nonnegative curvature operator is rigid. (This was proven in dimensions 2 and 3 by Hamilton [10] and Perelman [20] respectively.) Theorem 1.2 offers further evidence that this result extends to higher dimensions. In fact, in the proof all we use about cohomogeneity one is a much weaker condition on $f$ we call rectifiability, which we will discuss in section 3. (Also recall that the work of Böhm and Wilking $[3$ implies that every compact shrinking gradient Ricci soliton with nonnegative curvature operator is a quotient of the round sphere.) For other recent results concerning the classification of gradient shrinking solitons, see 8, 17, 18, 22, 24, 26.

The famous Bryant soliton (see [11) and the examples in [5] show that there are nonrigid rotationally symmetric steady and expanding gradient solitons with positive curvature operator.

\section{KILLING FIELDS ON GRADIENT SOLITONS}

In this section we establish a splitting theorem involving Killing fields on a gradient soliton which leads to Theorem[1.1. The main observation is the following.

Proposition 1. If $X$ is a Killing field on a gradient soliton, then $\nabla D_{X} f$ is parallel. Moreover, if $\lambda \neq 0$ and $\nabla D_{X} f=0$, then also $D_{X} f=0$.

Proof. We have that $L_{X} g=0$; thus $L_{X}$ Ric $=0$ and hence

$$
\begin{aligned}
0 & =L_{X} \operatorname{Hess} f \\
& =\operatorname{Hess} L_{X} f \\
& =\operatorname{Hess} D_{X} f .
\end{aligned}
$$

This proves the first claim.

Next note that if $\nabla D_{X} f=0$, then $D_{X} f$ is constant. Thus $f \circ \gamma_{X}(t): \mathbb{R} \rightarrow \mathbb{R}$ is onto if $\gamma_{X}$ is an integral curve for $X$ and $D_{X} f$ doesn't vanish.

On the other hand recall that the soliton equation implies that

$$
\text { scal }+|\nabla f|^{2}-2 \lambda f=\text { const. }
$$

So if the scalar curvature is bounded, we see that $f$ must be bounded from either below or above, and hence $D_{X} f=0$.

This shows that either $D_{X} f=0$ or the metric splits off a Euclidean factor. One might worry that the soliton structure may not also split; however, the next lemma shows that this is not an issue. 
Lemma 2.1. If a gradient soliton splits $(M, g)=\left(M_{1} \times M_{2}, g_{1}+g_{2}\right)$ as a Riemannian product, then $f\left(x_{1}, x_{2}\right)=f_{1}\left(x_{1}\right)+f_{2}\left(x_{2}\right)$ also splits in such a way that each $\left(M_{i}, g_{i}, f_{i}\right)$ is a soliton

$$
\operatorname{Ric}_{g_{i}}+\operatorname{Hess} f_{i}=\lambda g_{i} .
$$

Proof. Use the $(1,1)$ version of the soliton equation

$$
\text { Ric }+\nabla \nabla f=\lambda I
$$

to see that the operator $E \rightarrow \nabla_{E} \nabla f$ preserves the manifold splitting as the Ricci curvature preserves the splitting. This can be used to first split the gradient $\nabla f$. To see how, use local coordinates $x^{j}$ such that $x^{1}, \ldots, x^{m}$ are coordinates on $M_{1}$ and $x^{m+1}, \ldots, x^{n}$ are coordinates on $M_{2}$. The splitting of the metric then implies that

$$
\nabla_{\partial_{i}} \partial_{j}=0
$$

if $i \leq m$ and $j \geq m+1$ or if $i \geq m+1$ and $j \leq m$. If we write $\nabla f=\alpha^{j} \partial_{j}$, then

$$
\begin{aligned}
\nabla_{\partial_{i}} \nabla f & =\nabla_{\partial_{i}} \alpha^{j} \partial_{j} \\
& =\left(\partial_{i} \alpha^{j}\right) \partial_{j}+\alpha^{j} \nabla_{\partial_{i}} \partial_{j} .
\end{aligned}
$$

If we assume that $i \leq m$, then

$$
\begin{aligned}
\nabla_{\partial_{i}} \nabla f & \in T M_{1}, \\
\alpha^{j} \nabla_{\partial_{i}} \partial_{j} & \in T M_{1},
\end{aligned}
$$

showing that $\partial_{i} \alpha^{j}=0$ for $j \geq m+1$. Similarly $\partial_{i} \alpha^{j}=0$ when $i \geq m+1$ and $j \leq m$. This shows that

$$
\nabla f=X_{1}+X_{2}
$$

where $X_{i}$ are vector fields on $M_{i}$. We then see that

$$
X_{i}=\nabla f_{i}
$$

where

$$
\begin{aligned}
& f_{1}\left(x_{1}\right)=f\left(x_{1}, q\right), \\
& f_{2}\left(x_{2}\right)=f\left(p, x_{2}\right)-f(p, q)
\end{aligned}
$$

for some fixed point $(p, q) \in M_{1} \times M_{2}$.

Note that the splitting of the metric implies that

$$
R(\cdot, \nabla f) \nabla f=R_{1}\left(\cdot, \nabla f_{1}\right) \nabla f_{1}+R_{2}\left(\cdot, \nabla f_{2}\right) \nabla f_{2} .
$$

So if, say, $M_{2}$ is flat, then the radial curvatures of $M$ and $M_{1}$ are the same.

This implies the reduction result alluded to above.

Corollary 2.2. If $X$ is a Killing field on a gradient soliton, then either $D_{X} f=0$ or we have an isometric splitting $M=N \times \mathbb{R}$ where $N$ is a gradient soliton with the same radial curvatures as $M$.

Intuitively, Corollary 2.2 says that if the metric of a gradient soliton has some symmetry, then the only way $f$ can break the symmetry is by splitting off a Gaussian factor. With this fact we can prove the result for homogeneous solitons.

Theorem 2.3. All homogeneous gradient solitons are rigid. 
Proof. In the case where the soliton is steady, this is a consequence of the scalar curvature being constant and hence $M$ is Ricci flat.

When the soliton is expanding or shrinking, split $M=N \times \mathbb{R}^{k}$ such that $N$ doesn't have any flat de Rham factors. If $G$ acts transitively on $M$, then it also acts transitively on each of the two factors, as isometries preserve the flat de Rham factor.

The previous lemma and corollary now tell us that all Killing fields on $N$ must leave $f_{1}$ invariant. Thus $N$ can't be homogeneous unless $f_{1}$ is trivial.

\section{ReCtifiability}

In this section we prove the result for cohomogeneity one and more general rectifiable gradient solitons.

We say that a function $u$ is rectifiable if it can be written as $u=h(r)$ where $r$ is a distance function. It is easy to check that a function is rectifiable if and only if its gradient $\nabla u$ has constant length on the level sets of $u$. We will say that a gradient soliton $(M, g, f)$ is rectifiable if the function $f$ is rectifiable on $(M, g)$.

It is easy to see that a gradient soliton with a cohomogeneity 1 group action that leaves $f$ invariant is rectifiable. Assume that $G$ is such an isometric group action. This gives us a distance function

$$
r: M \rightarrow M / G \subset \mathbb{R}
$$

(locally if $G$ is noncompact) and $f=h(r)$ as $f$ is constant on the orbits of the action. Similarly the scalar curvature is also rectifiable with respect to $r$.

We note the following interesting properties of rectifiable solitons.

Proposition 2. If $(M, g, f)$ is a rectifiable gradient soliton with $f=h(r)$, then scal, $\Delta f$, and $\Delta r$ are also rectifiable. In particular, $\operatorname{Ric}(\nabla f, \nabla f)=0$ if and only if $(M, g)$ has constant scalar curvature.

Proof. If $f$ is rectifiable, then $|\nabla f|$ is also rectifiable, so the equation

$$
\text { scal }+|\nabla f|^{2}-2 \lambda f=\text { const }
$$

implies that the scalar curvature is rectifiable.

Tracing the soliton equation then gives

$$
\mathrm{scal}=\lambda n-\Delta f,
$$

so $\Delta f$ is rectifiable. Since $f$ is rectifiable we can write

$$
\Delta f=h^{\prime \prime}(r)+h^{\prime}(r) \Delta r .
$$

Thus $\Delta f$ being rectifiable implies that $\Delta r$ is also rectifiable.

Now since scal and $f$ are rectifiable, $\nabla$ scal $=\operatorname{Ric}(\nabla f)$ is proportional to $\nabla f$, proving the last statement.

The main result from 21] now shows that a rectifiable gradient soliton is rigid if and only if it is radially flat. We note that, in the case of cohomogeneity one, radial flatness, even without the soliton equation, is already quite restrictive. 
Theorem 3.1. A radially flat cohomogeneity 1 space coming from a compact action is a flat bundle.

Proof. Let $r: M \rightarrow \mathbb{R}$ be the distance function coming from the quotient $M \rightarrow$ $M / G$. It is smooth except at the singular orbits. The singular orbits correspond to the minima and/or maxima of $r$ if they exist.

Let $S_{r}=\nabla \nabla r$; then

$$
\nabla_{\nabla r} S_{r}+S_{r}^{2}=0 .
$$

This means that $S_{r}$ is completely determined by the singular orbits, where $S_{r} \rightarrow 0$ on vectors tangent to a singular orbit and $S_{r} \rightarrow \infty$ on vectors normal to the singular orbit and perpendicular to $\nabla r$.

If there are no singular orbits, then $S_{r}=0$ is the only possibility, as all other solutions blow up in finite time going forwards or backwards. Thus the space splits.

If $r$ has a minimum set, then solutions that start out being zero stay zero, while the other solutions that start out being $\infty$ decay to zero. As they never become zero, the space is noncompact. We see that the space must then be a flat bundle $N \times_{\Gamma} \mathbb{R}^{k}$ where $N / \Gamma$ is the singular orbit.

We now turn our attention to proving rigidity for rectifiable shrinking solitons with nonnegative radial curvature.

Proposition 3. Let $(M, g)$ be a Riemannian manifold and $r: M \rightarrow[0, \infty)$ a proper distance function that is smooth outside a compact set. If $\sec (E, \nabla r) \geq 0$, then $r$ is convex outside a compact set.

Proof. Define $S_{r}=\nabla \nabla r$ and use the fact that it solves the equation

$$
\nabla_{\nabla r} S_{r}=-S_{r}^{2}-R(\cdot, \nabla r) \nabla r .
$$

As $E \rightarrow R(E, \nabla r) \nabla r$ is assumed to be nonnegative, we see that if $S_{r}$ has a negative eigenvalue somewhere, then it will go to $-\infty$ before $r$ reaches infinity. This contradicts the assumption that $r$ is smooth.

Lemma 3.2. Let $(M, g, f)$ be a noncompact nontrivial shrinking gradient soliton with rectifiable and proper $f$. If the radial curvatures $\sec (E, \nabla f)$ are nonnegative, then $f$ is convex at infinity.

Proof. Since $f$ is rectifiable, $f=h(r)$ where $r: M \rightarrow[0, \infty)$ is a distance function that is smooth outside a compact set. Since $f$ and $r$ have proportional gradients, i.e., $\nabla f=h^{\prime} \nabla r$, our curvature assumption guarantees that $r$ is convex at infinity.

First note that the equation

$$
\text { scal }+|\nabla f|^{2}-2 \lambda f=\text { const }
$$

shows that $|\nabla f| \rightarrow \infty$ as scal is bounded and $f$ is proper, i.e., $|f| \rightarrow \infty$. In particular $h^{\prime}>0$ outside a compact set. 
Define $S_{f}=\nabla \nabla f$ and $S_{r}=\nabla \nabla r$; they are related by

$$
\begin{aligned}
S_{f} & =\nabla \nabla f \\
& =h^{\prime \prime} d r \otimes \nabla r+h^{\prime} \nabla \nabla r \\
& =h^{\prime \prime} d r \otimes \nabla r+h^{\prime} S_{r} .
\end{aligned}
$$

The soliton equation shows that

$$
\operatorname{Ric}(\nabla r, \nabla r)+h^{\prime \prime}=\lambda .
$$

Since Ric $(\nabla r, \nabla r)$ is nonnegative, this gives $h^{\prime \prime} \leq \lambda$.

Next we claim that Ric $(\nabla r) \rightarrow 0$ as $r \rightarrow \infty$. This follows from the formula

$$
\begin{aligned}
\operatorname{Ric}(\nabla r) & = \pm \frac{\operatorname{Ric}(\nabla f)}{|\nabla f|} \\
& = \pm \frac{1}{2} \frac{\nabla \text { scal }}{|\nabla f|}
\end{aligned}
$$

where we note that $\nabla$ scal is bounded and $|\nabla f| \rightarrow \infty$ at infinity.

Thus

$$
S_{f}(\nabla r)=h^{\prime \prime} \nabla r \sim \lambda \nabla r
$$

at infinity. This proves that outside some large compact set, $h^{\prime \prime} \geq \lambda / 2$ and $h^{\prime}>0$. Thus $f$ is convex outside a compact set.

Theorem 3.3. A complete, noncompact, rectifiable, shrinking gradient soliton with nonnegative radial sectional curvature and nonnegative Ricci curvature is rigid.

Proof. Let $f=h(r)$. Since we have a shrinking gradient Ricci soliton with bounded nonnegative curvature, $f$ is proper [20]. Therefore, the previous lemmas show that $f$ and $r$ are proper and convex outside a compact set. This implies that Ric $\leq \lambda g$ outside a compact set. Define $\Delta_{f}=\Delta-D_{\nabla f}$ to be the $f$-Laplacian, then (see [21])

$$
\Delta_{f} \text { scal }=\operatorname{tr}(\operatorname{Ric} \circ(\lambda I-\operatorname{Ric})) .
$$

So Ric $\leq \lambda g$ outside a compact set implies

$$
\Delta_{f} \text { scal } \geq 0
$$

outside a set $\Omega_{R}=\{x \in M: r \leq R\}$. We also know that scal is increasing along gradient curves for $\nabla f$, as

$$
D_{\nabla f} \text { scal }=2 \operatorname{Ric}(\nabla f, \nabla f) \geq 0 .
$$

If

then the function

$$
s_{R}=\min _{p \in \partial \Omega_{R}} \operatorname{scal}_{p},
$$

$$
u=\max \left\{\mathrm{scal}, s_{R}\right\}
$$

satisfies

$$
\Delta_{f} u \geq 0 .
$$

From Theorem 4.2 in 22 it follows that $u$ is constant (also see 25]). This shows that scal $=s_{R}$ on $M-\Omega_{R}$. Since $(M, g)$ is analytic (see [2]) the scalar curvature is constant on all of $M$. This in turn shows that Ric $(\nabla f, \nabla f)=0$ everywhere, and hence $\sec (E, \nabla f) \geq 0$ implies that $(M, g)$ is radially flat. The main theorem from 21] then shows that $M$ is rigid. 


\section{REFERENCES}

1. Paul Baird and Laurent Danielo. Three-dimensional Ricci solitons which project to surfaces. J. Reine Angew. Math. 608: 65-91, 2007. MR2339469 (2008f:53086)

2. Shigetoshi Bando. Real analyticity of solutions of Hamilton's equation. Math Z. 195: 93-97, 1987. MR 888130 (88i:53073)

3. Christoph Böhm and Burkhard Wilking. Manifolds with positive curvature operators are space forms. Ann. of Math. (2) 167(3): 1079-1097, 2008.

4. Huai-Dong Cao. Existence of gradient Kähler-Ricci solitons. In Elliptic and parabolic methods in geometry (Minneapolis, MN, 1994), pages 1-16. A K Peters, Wellesley, MA, 1996. MR 1417944 (98a:53058)

5. Huai-Dong Cao. Limits of solutions to the Kähler-Ricci flow. J. Differential Geom. 45(2): 257-272, 1997. MR1449972 (99g:53042)

6. Bennet Chow, Peng Lu, and Lei Ni. Hamilton's Ricci flow. Graduate Studies in Mathematics 77, Amer. Math. Soc., Providence, RI; Science Press, New York, 2006. MR 2274812 (2008:53068)

7. Andrew Dancer and Mckenzie Wang. On Ricci Solitons of cohomogeneity one. arXiv:math/0802.0759.

8. Manolo Eminenti, Gabriele La Nave, and Carlo Mantegazza. Ricci Solitons - the Equation Point of View. Manuscripta Math. 127(3): 345-367, 2008.

9. Mikhail Feldman, Tom Ilmanen, and Dan Knopf. Rotationally symmetric shrinking and expanding gradient Kähler-Ricci solitons. J. Differential Geom. 65(2): 169-209, 2003. MR 2058261 (2005e:53102)

10. Richard Hamilton. The Ricci flow on surfaces. In Mathematics and General Relativity, Contemporary Mathematics 71, pages 237-262. Amer. Math. Soc., Providence, RI, 1988. MR 954419 (89i:53029)

11. Thomas Ivey. New examples of complete Ricci solitons. Proc. Amer. Math. Soc. 122(1): 241-245, 1994. MR.1207538 (94k:53057)

12. Norihito Koiso. On rotationally symmetric Hamilton's equation for Kähler-Einstein metrics. In Recent Topics in Differential and Analytic Geometry, Adv. Stud. Pure Math. 18, pages 327-337. Academic Press, Boston, MA, 1990. MR1145263 (93d:53057)

13. Brett Kotschwar. On rotationally invariant shrinking gradient Ricci solitons. Pacific J. Math. 236(1): 73-88, 2008.

14. Jorge Lauret. Ricci soliton homogeneous nilmanifolds. Math. Ann. 319: 715-733, 2001. MR 1825405 (2002k:53083)

15. John Lott. On the long-time behavior of type-III Ricci flow solutions. Math. Ann. 339(3): 627-666, 2007. MR2336062(2008i:53093)

16. Aaron Naber. Noncompact shrinking 4-solitons with nonnegative curvature. arXiv:math.DG/0710.5579.

17. Lei Ni. Ancient solutions to Kähler Ricci flow. Math. Research Letters 12: 633-653, 2005. MR2189227 (2006i:53097)

18. Lei $\mathrm{Ni}$ and Nolan Wallach. On a classification of the gradient shrinking solitons. Math. Research Letters 15(5): 941-955, 2008.

19. Lei Ni and Nolan Wallach. On 4-dimensional gradient shrinking solitons. Internat. Math. Res. Notices, vol. 2008, rnm152, 13 pp.

20. G. Ya. Perelman. Ricci flow with surgery on three manifolds. arXiv: math.DG/0303109.

21. Peter Petersen and William Wylie. Rigidity of gradient Ricci solitons. arXiv:math.DG/0710.3174. To appear in Pacific J. Math.

22. Peter Petersen and William Wylie. On the classification of gradient Ricci solitons. arXiv:math.DG/0712.1298.

23. X.J. Wang and X.H. Zhu. Kähler-Ricci solitons on toric manifolds with positive first Chern class. Adv. Math. 188(1): 87-103, 2004. MR2084775 (2005d:53074)

24. Brian Weber. Convergence of compact Ricci solitons. arXiv:math.DG/0804.1158.

25. Guofang Wei and William Wylie. Comparison geometry for the Bakry-Emery Ricci tensor. arXiv:math.DG/0706.1120. 
26. William Wylie. Complete shrinking Ricci solitons have finite fundamental group. Proc. Amer. Math. Soc. 136(5): 1803-1806, 2008. MR 2373611

27. Bo Yang. A characterization of Koiso's typed solitons. arXiv:math.DG/0802.0300.

Department of Mathematics, University of California, los Angeles, 520 Portola Plaza, los Angeles, California 90095

E-mail address: petersen@math.ucla.edu

$U R L:$ http://www.math.ucla.edu/ ${ }^{\sim}$ petersen

Department of Mathematics, University of California, Los Angeles, 520 Portola Plaza, Los Angeles, California 90095

Current address: Department of Mathematics, David Rittenhouse Laboratory, University of Pennsylvania, 209 South 33rd Street, Philadelphia, Pennsylvania 19104-6395

E-mail address: wylie@math.upenn.edu

URL: http://www.math. upenn.edu/ ${ }^{\sim}$ wylie 